\title{
CT and MR Imaging Findings in the Joubert Syndrome, a "Ciliopathy"
}

\author{
Kaveh Akbari'1, Christine M. Fellner'1, Daniel Flöry'1, Franz A. Fellner1,2* \\ ${ }^{1}$ Institute of Radiology, Kepler University Clinic, Medical Faculty of the Johannes Kepler University, Linz, Austria \\ ${ }^{2}$ Medical Faculty of the Friedrich-Alexander-University Erlangen-Nürnberg, Erlangen, Germany \\ Email: franz.fellner@akh.linz.at
}

Received 18 September 2015; accepted 12 October 2015; published 15 October 2015

Copyright $\odot 2015$ by authors and Scientific Research Publishing Inc.

This work is licensed under the Creative Commons Attribution International License (CC BY).

http://creativecommons.org/licenses/by/4.0/

(c) () Open Access

\section{Abstract}

A 7-year-old boy presented with cerebellar ataxia with reduced tonicity, deficits of the fine and gross motor coordination skills and vestibular stimulus processing, as well as significantly delayed language development. MR imaging showed the so-called "molar tooth sign", which was highly pathognomonic for the Joubert-Syndrome-an inherited cerebellar ataxia with a variety of clinical symptoms-and related entities. It is caused by a complex malformation of the cerebellar vermis and the midbrain. The cerebellar vermis is hypoplastic or completely absent; at the same time, the superior cerebellar peduncles are thickened. There is a lack of normal decussation of the fiber tracts in mesencephalon, which follow an abnormal horizontal course, as well as a lack of the decussation of the corticospinal fiber tracts in the caudal medulla oblongata and deformity of the 4th ventricle. Clinically, the triad of cerebellar ataxia, developmental retardation, and abnormal eye movements is indicating a related syndrome of this spectrum. The appearance of the involved children is characterized by dysmorphic facial features with epicanthus, broad nose bridge, low set ears and typically triangularly shaped and opened mouth. The diagnosis is usually made by imaging and clinical findings. Recently, advantages were made in genetic research on the Joubert syndrome and interesting findings published about diffusion tensor imaging and tractography. However, standard MR imaging, applying an adequate imaging protocol including sequences with excellent $\mathrm{T} 1$ contrast and 3D imaging with isotropic spatial resolution allowing reconstructions in all orientations, remains an essential tool for making this diagnosis.

\section{Keywords}

Joubert Syndrome, Ciliopathy, Cerebellar Ataxia, Molar Tooth Sign, Magnetic Resonance (MR) Imaging

${ }^{*}$ Corresponding author. 


\section{Introduction}

The Joubert syndrome, an inherited cerebellar ataxia with a variety of clinical symptoms, is an autosomal recessive disease, which is characterized by a congenital midbrain-hindbrain malformation involving the cerebellum and brain stem resulting in a characteristical "molar tooth sign” and variable organ involvement. It has been first described in 1969 by the Canadian neurologist Marie Joubert [1].

Some of the patients have a mutation on the long arm of chromosome 9 (9q34.3), and the prevalence is about $1: 100,000$ [2]. So far, other forms with different gene mutations have been described, which are subsumed as cerebello-oculo-renal syndromes (e.g. Arima, Senior-Löken and COACH syndromes), as ocular and renal, and even hepatic changes can occur in addition to cerebellar alterations.

From a pathogenetic point of view, in the meanwhile, dysfunction of the "primary cilium”, a subcellular organelle, has been identified as the basis of this genetically and clinically heterogeneous group of disorders, therefore called “ciliopathies” [3], including Joubert syndrome (JS) and related disorders (JSRD), Meckel Gruber syndrome (MKS), and Bardet-Biedl syndrome (BBS) [4].

Clinically, the Joubert syndrome presents a variety of symptoms. Due to the malformation of the cerebellar vermis, there is cerebellar ataxia with reduced tonicity. Often an irregular breathing pattern and nystagmus are seen. Additionally, delayed motoric and language development with moderate mental retardation is usually present. As associated organ manifestations, cystic kidneys or liver fibrosis can appear. Also a lot of more malformations have been described, such as dysplasia of the retina with developing of colobomas, associated congenital heart failures, polydactylia and meningoencephalocele or hydrocephalus.

The appearance of the involved children is characterized by dysmorphic facial features with epicanthus, broad nose bridge, low set ears and typically triangularly shaped and opened mouth.

\section{Case Report}

We report a 7-year-old boy, who was referred to the department of paediatric medicine just before school enrolment due to mental retardation. In the initial neurological examination, he presented especially with a cerebellar ataxy with reduced tonicity. Further findings included deficits of the fine and gross motor coordination skills and the vestibular stimulus processing. Also the language development was significantly delayed, he was able to speak a few words only at the time of the testing.

MR examination of the cerebrum was performed, revealing aplasia of the cerebellar vermis (Figure 1), as well as showing the peculiar finding of a so called "molar tooth sign", which is characteristic for the Joubert-Syndrome and related entities (Figure 2).

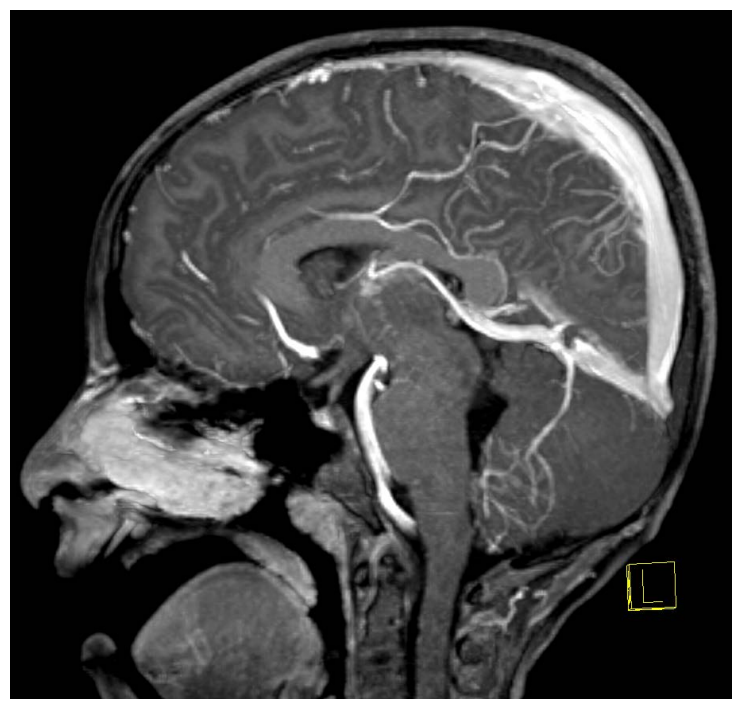

(a)

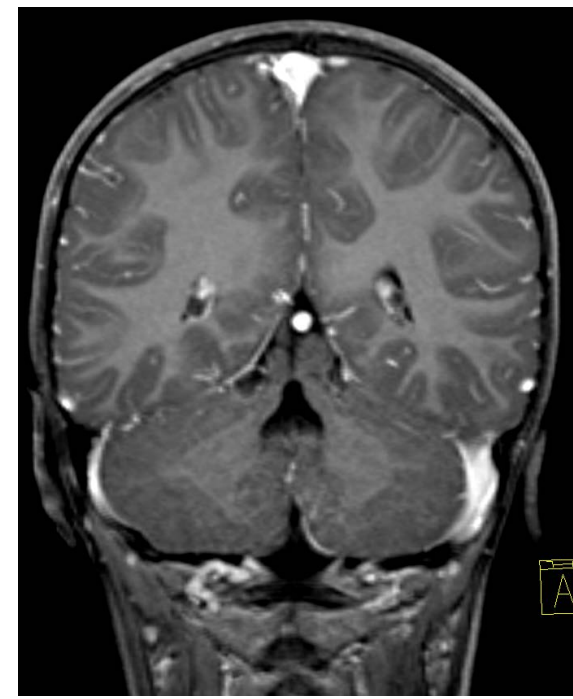

(b)

Figure 1. Gd-enhanced T1-w 3D gradient-echo data set (3D-MPRAGE) with sagittal (a) and coronal (b) reconstructions shows clearly an infratentorial midline abnormality in the sense of aplasia of the cerebellar vermis. 


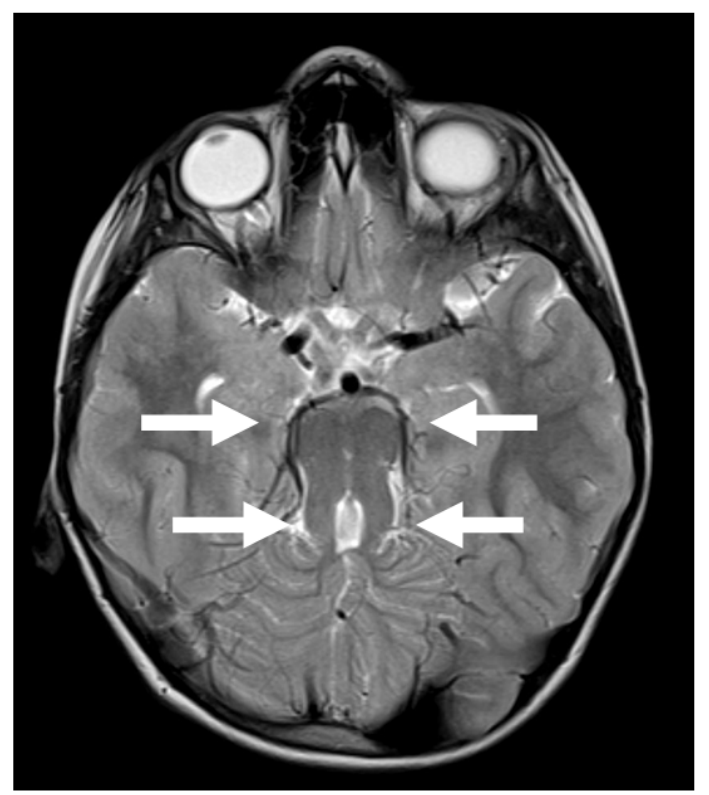

Figure 2. Transverse T2-weighted TSE sequence: congenital midbrain-hindbrain malformation involving the cerebellum and brain stem results in the typical molar tooth like deformation of the mesencephalon, called "molar tooth sign” (arrows).

It is caused by the lack of normal decussation of superior cerebellar peduncular fiber tracts, which are thickened and follow an abnormal horizontal course as a result (Figure 3).

Furthermore, the absence of crossing fibers leads to a deepening of the interpeduncular cistern. The 4th ventricle is typically widened and shows an umbrella-like structure in the transverse orientation (Figure 4). There were no abnormalities concerning the cerebellar hemispheres or the supratentorial brain.

Abdominal ultrasound examination ruled out an associating involvement of the liver or the kidneys. Ophthalmologic examination confirmed nystagmus, whereas a retinal dystrophy did not exist.

Finally, neuropaediatric evaluation followed to quantify the motoric and language deficits and to plan the appropriate ergotherapeutical and logopaedic therapy. The patient was enrolled in a special school for handicapped children.

\section{Discussion}

The Joubert syndrome and related cerebello-oculo-renal diseases show characteristic findings in imaging methods CT and especially MR. A complex malformation of the cerebellar vermis and the midbrain exists in all cases. The cerebellar vermis is typically hypoplastic or completely absent, at the same time, the superior cerebellar peduncula are thickened. There is lack of normal decussation of the fiber tracts in the mesencephalon, which follow an abnormal horizontal course, as well as a lack of the decussation of the coricospinal fiber tracts in the caudal medulla oblongata and deformity of the 4th ventricle. These changes lead to the highly pathognomonic "molar tooth sign" in the imaging, which is evident in about 85\% of the patients with Joubert syndrome [5] [6].

The lack of the decussation of superior cerebellar peduncular fiber tracts, as well as the decussation of the corticospinal fiber tracts in the caudal medulla oblongata were shown in recent studies by Diffusion Tensor Imaging and Fiber Tracking. The examination results, however, could not help to differentiate genetic subtypes of the Joubert syndrome [7]. The benefit of these technics in the clinical imaging is not definitively determined and remains a subject of ongoing research.

Clinically, especially the triad of cerebellar ataxia, developmental retardation, and abnormal eye movements is indicating a related syndrome of this spectrum. The diagnosis is usually made by imaging and clinical findings. The patient described in this paper had a classical Joubert syndrome, as he revealed no further organ manifestations. If clinically suspected, an MR examination of the cerebrum should follow. Basically, the "molar tooth 


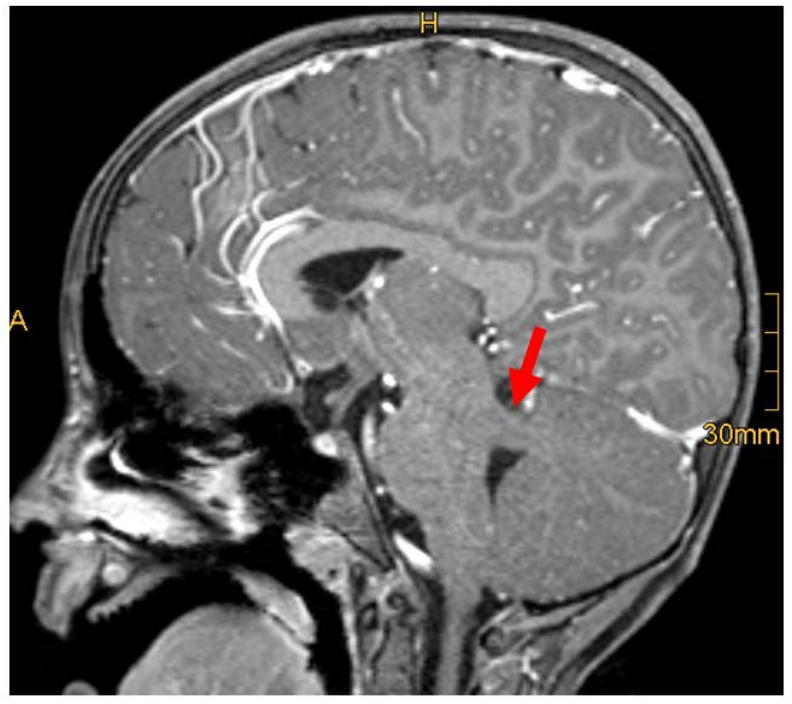

Figure 3. T1-weighted Gd-enhanced 3D-MPRAGE sequence: In the infratentorial area the superior cerebellar peduncular fiber tracts are thickened and therefore, the superior cerebellar peduncles follow an abnormal horizontal course (arrow).

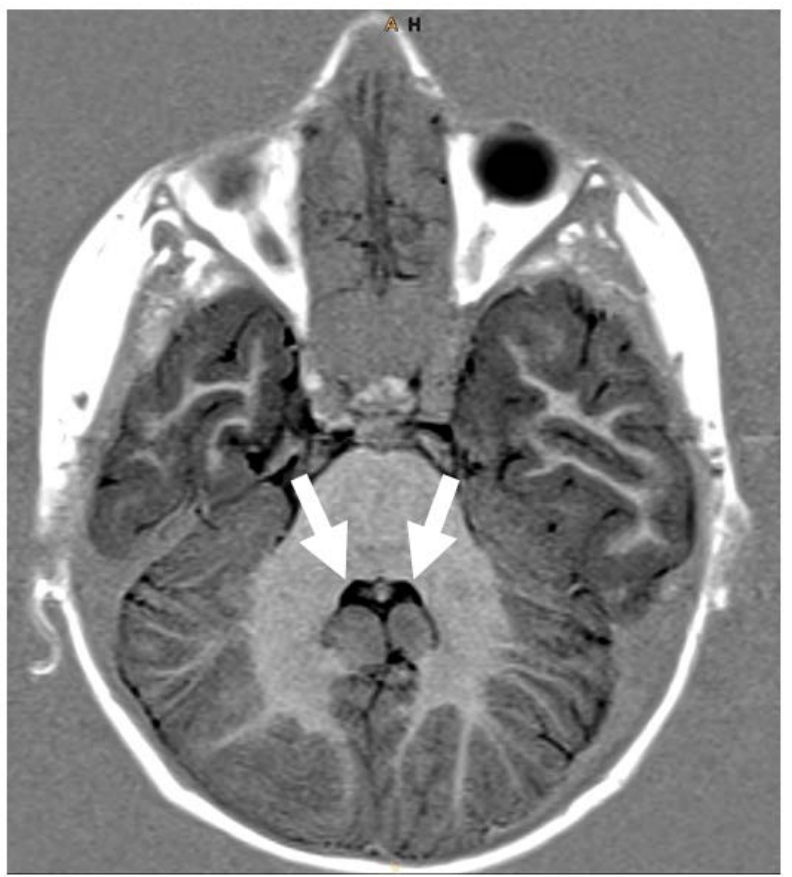

Figure 4. Transverse T1-weighted 2D inversion recovery sequence: In the Joubert syndrome there is a characteristic deformity of the brain stem in the sense of a strange, but characteristic umbrella-like configuration of the 4 th ventricle (arrows).

sign” is also seen on CT in most instances, but MR is more sensitive and allows also the evaluation of additional cerebral findings.

Differential diagnosis includes hypoplasia of the cerebellar vermis related with a Dandy-Walker-Syndrome or a rhombencephalosynapsis. Both syndromes can be differentiated from the Joubert syndrome by adequate imaging. The Dandy-Walker-Malformation shows a big cystic formation in the posterior fossa, which is not evi- 
dent in patients with Joubert syndrome. Rhombencephalosynapsis shows a characteristic fusion of the cerebellar hemispheres, the fissure, which is typical for Joubert syndrome, is missing.

MR imaging as the essential tool for making the diagnosis should be performed with an adequate protocol including sequences with excellent T1 contrast (e.g. inversion recovery) and 3D imaging (e.g. T1-weighted 3D gradient-echo data sets) with isotropic resolution allowing high quality of reformations in all spatial orientations omitting separate (time-consuming) measurements in different orientations.

After diagnosis of Joubert syndrome in our patient, adequate therapy was started immediately. After neuropaediatric evaluation to quantify motoric and speech deficits, appropriate ergotherapeutical and logopaedic therapy was planned. Consecutively, the patient was enrolled in a special school for handicapped children.

Only symptomatic therapy is available for patients with the Joubert syndrome. Therapy management should include annual monitoring of the liver and renal function, as well as continuous ophthalmologic examinations. Particularly important is also an adequate ergotherapeutical and logopaedical support. In case of behaviour disorder, a neuropsychologic support could be indicated. A genetic counselling of the affected families should be provided, as prenatal DNA tests exist.

\section{Conclusion}

Despite recent advantages in genetic research on the Joubert syndrome and interesting findings applying diffusion tensor imaging and tractography, we have seen that standard MR imaging remains the essential diagnostic tool in the case of Joubert syndrome, on condition that an adequate imaging protocol is applied. It is essential to include sequences with excellent T1 contrast as well as 3D imaging (T1- or T2-weighted) with isotropic spatial resolution allowing reconstructions in all orientations.

\section{References}

[1] Joubert, M., Eisenring, J.J., Robb, J.P. and Anderman, F. (1969) Familial Agenesis of the Cerebellar Vermis. A Syndrome of Episodic Hyperpnea, Abnormal Eye Movements, Ataxia, and Retardation. Neurology, 19, 813-825. http://dx.doi.org/10.1212/WNL.19.9.813

[2] Saar, K., Al-Gazali, L., Sztriha, L., Rueschendorf, F., Nur-E-Kamal, M., Reis, A. and Bayoumi, R. (1999) Homozygosity Mapping in Families with Joubert Syndrome Identifies a Locus on Chromosome 9q34.3 and Evidence for Genetic Heterogeneity. American Journal of Human Genetics, 65, 1666-1671. http://dx.doi.org/10.1086/302655

[3] Romani, M., Micalizzi, A. and Valente, E.M. (2013) Joubert Syndrome: Congenital Cerebellar Ataxia with the Molar Tooth. Lancet Neurology, 12, 894-905. http://dx.doi.org/10.1016/S1474-4422(13)70136-4

[4] Guo, J., Higginbotham, H., Li, J., Nichols, J., Hirt, J., Ghukasyan, V. and Anton, E.S. (2015) Developmental Disruptions Underlying Brain Abnormalities in Cilipathies. Nature Communications, 6, 7857. http://dx.doi.org/10.1038/ncomms8857

[5] Spampinato, M.V., Kraas, J., Maria, B.L., Walton, Z.J. and Rumboldt, Z. (2008) Absence of Decussation of the Superior Cerebellar Peduncles in Patients with Joubert Syndrome. American Journal of Medical Genetics Part A, 146, 1389-1394. http://dx.doi.org/10.1002/ajmg.a.32282

[6] Maria, B.L., Quisling, R.G., Rosainz, L.C., Yachnis, A.T., Gitten, J., Dede, D. and Fennell, E. (1999) Molar Tooth Sign in Joubert Syndrome: Clinical, Radiologic, and Pathologic Significance. Journal of Child Neurology, 14, 368376. http://dx.doi.org/10.1177/088307389901400605

[7] Poretti, M., Meoded, A., Rossi, A., Raybaud, C. and Huisman, T.A. (2013) Diffusion Tensor Imaging and Fiber Tractography in Brain Malformations. Pediatric Radiology, 1, 28-54. http://dx.doi.org/10.1007/s00247-012-2428-9 